\title{
Variaciones en el patrón espacial a pequeña escala de los componentes de la costra biológica en un ecosistema mediterráneo semiárido
}

\author{
Small-scale spatial patterns of biological crusts in a Mediterranean semi-arid \\ ecosystem
}

FERNANDO T. MAESTRE

Departamento de Ecología, Universidad de Alicante, Apartado de Correos 99, 03080 Alicante, España; e-mail: FT.Maestre@ua.es

\begin{abstract}
RESUMEN
La costra biológica constituye una de las propiedades edáficas más importantes en los ecosistemas semiáridos, donde juegan un importante rol en su funcionamiento y dinámica. En los últimos años se ha producido un notable aumento en el conocimiento de la ecofisiología, dinámica y taxonomía de los constituyentes de este tipo de costras, pero pocos estudios hasta la fecha han evaluado los cambios que la vegetación introduce en su patrón espacial a pequeña escala. Con el objetivo de avanzar en el conocimiento sobre las interacciones entre los componentes de la costra biológica y las plantas vasculares, en este artículo se evalúan los efectos del microambiente proporcionado por Stipa tenacissima L. en la distribución espacial de los líquenes foliáceos y los musgos, en estepas semiáridas del SE de España. El principal objetivo de estudio es determinar si el microambiente proporcionado por $S$. tenacissima promueve la aparición de cambios en el patrón individual de estos componentes de la costra biológica por separado y en su covariación espacial. Para alcanzar este objetivo se ha utilizado el análisis espacial mediante índices de distancia ("spatial analysis by distance indices", SADIE), mediante el cual se detectó un patrón de distribución agregado en los líquenes foliáceos y los musgos, pero el microambiente proporcionado por S. tenacissima sólo introdujo cambios significativos en el patrón espacial de los primeros. No se encontró un efecto significativo del microambiente proporcionado por S. tenacissima en la covariación espacial entre los líquenes foliáceos y los musgos. Los resultados obtenidos muestran cómo $S$. tenacissima es capaz de modificar la distribución espacial a pequeña escala de los componentes de la costra biológica y sugieren la facilitación entre esta especie y los líquenes foliáceos.
\end{abstract}

Palabras clave: costra biológica, estepa, líquenes foliáceos, musgos, Stipa tenacissima.

\begin{abstract}
Biological crusts are a common feature of soil surface in arid and semiarid ecosystems, where they play a major role in ecosystem functioning and dynamics. In recent years, there has been an increasing interest in the ecophysiology, taxonomy, and dynamics of these crusts, but little is known about the effect of vascular plants on their small-scale spatial distribution. With the aim to increase our understanding about the interactions between biological crusts and vegetation in semi-arid areas, in this paper I evaluate the spatial pattern and interaction of two biological crusts components, foliose lichens and mosses, at two microsites in semi-arid Stipa tenacissima L. steppes of SE Spain. The aim of this paper was to determine if the microsite provided by S. tenacissima tussocks promoted changes in the individual patterns and in the spatial covariation of these crustal components. I used spatial analysis by distance index (SADIE) to explore the individual patterns and the spatial relationships between them. SADIE detected a significant clumped pattern in the spatial distribution of both species, but the effect of S. tenacissima tussocks promoted changes in the spatial pattern only in the foliose lichens. I did not find any microsite effect on the spatial relationships between the foliose lichens and the mosses. The results showed that the microenvironment provided by S. tenacissima is able to modify the small-scale spatial pattern of foliose lichens in semi-arid steppes, and suggested the presence of facilitation between S. tenacissima and these lichens.
\end{abstract}

Key words: biological crusts, foliose lichens, mosses, Stipa tenacissima, steppe.

\section{INTRODUCCIÓN}

La capacidad que tienen las plantas de alterar las condiciones ambientales en sus alrededores y modificar los flujos de recursos y energía dentro de los ecosistemas ha sido ampliamente reconoci- da (e.g., Binkley \& Giardina 1998, Schlesinger \& Pilmanis 1998). Esta facultad de la vegetación cobra especial relevancia en las zonas áridas y semiáridas, donde predominan formaciones vegetales abiertas en las que alternan manchas de vegetación dispuestas en una matriz de suelo 
desnudo (Puigdefábregas \& Sánchez 1996). Bajo estas condiciones, el patrón espacial de la vegetación va a jugar un papel fundamental en la distribución de las propiedades edáficas (Cross \& Schlesinger 1999), la microtopografía (Puigdefábregas \& Sánchez 1996) y el microclima (Breshears et al. 1998). Los cambios inducidos por la vegetación en la distribución de estas variables originan la formación de "islas de recursos" (Reynolds et al. 1999) en las zonas vegetadas, que incrementan la productividad y diversidad de los ecosistemas semiáridos (Aguiar \& Sala 1999).

La formación, mantenimiento y dinámica de las "islas de recursos" depende de los flujos de agua, sedimentos, nutrientes y semillas procedentes de las zonas desnudas (Aguiar \& Sala 1999). Una de las propiedades edáficas superficiales que van a jugar un importante papel en la generación de estos flujos es la costra biológica formada por bacterias, cianobacterias, algas, musgos y líquenes (West 1990, Eldridge et al. 2000, Belnap et al. 2001). Esta costra constituye también un importante aporte de carbono y nitrógeno al suelo, incrementa su estabilidad y la protege frente a la acción erosiva de la lluvia y el viento (Belnap et al. 2001). Asímismo, puede influenciar el establecimiento (Zaady et al. 1997, Prasse \& Bornkamm 2000), estado nutricional (Harper \& Belnap 2001) e hídrico (DeFalco et al. 2001) de las plantas vasculares. Dado el importante papel que la costra biológica tiene en el funcionamiento de los ecosistemas semiáridos (West 1990, Belnap et al. 2001), en los últimos años se ha producido un notable aumento en el conocimiento de la ecofisiología, dinámica, taxonomía y distribución de sus constituyentes (e.g., Lange et al. 1992, Eldridge 1996, Eldridge \& Tozer 1997). Diversos estudios sugieren que los cambios microclimáticos y edáficos promovidos por el desarrollo de la vegetación pueden favorecer el desarrollo de determinados componentes de la costra biológica, que de otra forma no podrían sobrevivir a las adversas condiciones climáticas presentes en las zonas desnudas (MartínezSánchez et al. 1994, Belnap et al. 2001). No obstante, pocos estudios hasta la fecha han evaluado los cambios que estas modificaciones tiene sobre la distribución a pequeña escala de los componentes de esta costra (Maestre et al. 2002) y ninguno de ellos ha utilizado técnicas de análisis espacial cuantitativas, a pesar de la importancia que estas herramientas han adquirido en las últimas décadas en el ámbito de la ecología vegetal (Dale 1999).

Una de las especies más importantes en la cuenca mediterránea es Stipa tenacissima L., gramínea perenne que forma comunidades esteparias que dominan el paisaje de las zonas semiáridas del sudoeste de Europa y el norte de África (Le Houérou 2001). El suelo existente debajo de la copa de S. tenacissima se caracteriza por presentar una menor compactación (Bochet et al. 1999) y una mayor porosidad (Puigdefábregas et al. 1999), tasa de infiltración (Cerdà 1997, Maestre et al. 2002) y contenido en materia orgánica (Puigedfábregas \& Sánchez 1996) que los espacios desnudos contiguos. La sombra producida por esta especie reduce la radiación PAR y la temperatura edáfica (Maestre et al. 2001) en comparación con las zonas sin vegetación. Todos estos cambios pueden facilitar (sensu Callaway 1995) el desarrollo de otras especies. Así, Sánchez (1995) observa la existencia de mayor cobertura de herbáceas en los primeros 0-10 cm de distancia a las macollas de $S$. tenacissima, mientras que Martínez-Sánchez et al. (1994) describen la presencia de numerosas especies de líquenes y musgos debajo de la copa de $S$. tenacissima que no se encuentran en las superficies desnudas adyacentes. Maestre et al. (2002) observan una mayor cobertura de musgos en las inmediaciones de $S$. tenacissima que en las zonas desnudas adyacentes, que presentaron una mayor cobertura de cianofíceas.

Con el objeto de avanzar en el conocimiento sobre las interacciones entre los componentes de la costra biológica y la vegetación en zonas semiáridas, en este artículo se evalúan los efectos del microambiente proporcionado por $S$. tenacissima en el patrón espacial a pequeña escala y en las interacciones en el espacio de dos de los componentes más importantes de dicha costra, los líquenes y los musgos, en estepas semiáridas del SE de España. En zonas semiáridas, la presencia de estos organismos se ve favorecida por el aumento de la humedad edáfica y la estabilidad del suelo (Eldridge \& Tozer 1997). La hipótesis que se maneja en este estudio es que las modificaciones microambientales promovidas por el desarrollo de S. tenacissima pueden modificar el patrón espacial de líquenes y musgos, así como su covariación espacial. Los principales objetivos de este estudio fueron (i) caracterizar el patrón espacial a pequeña escala de los musgos y líquenes y (ii) evaluar si el microambiente proporcionado por $S$. tenacissima puede promover la aparición de cambios en el patrón individual de estas especies por separado y en la variación conjunta en el espacio. Para alcanzar estos objetivos se ha utilizado una herramienta de análisis espacial desarrollada recientemente, el método SADIE ("spatial analysis by distance indices", Perry 1998, Perry et al. 1999). Esta técnica se ha utilizado con éxito para la caracterización de la 
distribución espacial de insectos (Winder et al. 2001), hongos patógenos de plantas (Turechek \& Madden 1999), virus vegetales (Perry 1998), malezas (Perry 1995), nemátodos (Perry 1998) y propiedades edáficas superficiales (Maestre \& Cortina 2002). Hasta la fecha ningún estudio ha utilizado este método para la caracterización del patrón espacial a pequeña escala de los componentes de la costra biológica, por lo que se evalúa también su aptitud para este fin.

\section{MATERIALES Y MÉTODOS}

\section{Área de estudio}

El estudio se llevó a cabo en tres parcelas experimentales localizadas en las cercanías de Sax, en la provincia de Alicante, SE de España (Colominas, $38^{\circ} 35^{\prime} \mathrm{N} 0^{\circ} 48^{\prime} \mathrm{O}$, altitud $640 \mathrm{~m}$; $\mathrm{La}$ Cueva, $38^{\circ} 35^{\prime} \mathrm{N} 0^{\circ} 48^{\prime} \mathrm{O}$, altitud $625 \mathrm{~m}$; La Torre, $38^{\circ} 34^{\prime} \mathrm{N} 0^{\circ} 48^{\prime} \mathrm{O}$, altitud $619 \mathrm{~m}$ ). Las tres parcelas se encuentran en zonas de orientación sudoeste y tienen una pendiente inferior a $8^{\circ}$. El clima es termomediterráneo semiárido, con una precipitación media anual de 315,4 mm (4 mm en agosto y $45 \mathrm{~mm}$ en octubre) y una temperatura media anual de $16,5^{\circ} \mathrm{C}\left(8,4{ }^{\circ} \mathrm{C}\right.$ en enero y $26,2^{\circ} \mathrm{C}$ en julio) para el período 1986-1995 (Maestre 2000). El contenido en materia orgánica de los suelos (0-5 cm de profundidad), calculado mediante la técnica de pérdida de masa por combustión a $550^{\circ} \mathrm{C}$ durante dos horas, oscila entre el 3,5 y $4,6 \%$ (Colominas $=3,50 \pm 0,38 ;$ La Cueva $=$ 4,55 $\pm 0,34$; La Torre $=3,64 \pm 0,24$; media \pm error estándar; $n=12)$, aunque las diferencias entre parcelas no son significativas (ANOVA de un factor; $\left.\mathrm{F}_{2,33}=3,15 ; \mathrm{P}=0.056\right)$. La vegetación consiste en una estepa de la asociación LapiedroMartinezii Stipetum tenacissimae (Rivas-Martínez \& Alcaraz 1972) y está dominada por $S$. tenacissima, pero incluye también especies arbustivas y arbóreas mediterráneas como Rosmarinus officinalis L., Globularia alypum L., Anthyllis cytisoides L. y Pinus halepensis Miller. Para la nomenclatura de las plantas vasculares se ha seguido a Mateo \& Crespo (2001).

\section{Especies utilizadas y adquisición de datos}

La morfología de los componentes de la costra biológica es fundamental a la hora de determinar su función ecológica en relación a procesos como la infiltración, erosión, retención de la humedad y resiliencia frente a las perturbaciones (Eldridge \& Rosentreter 1999, Belnap et al. 2001). Debido a la dificultad que plantea la determinación de las especies que componen la costra biológica in situ, y a la relación entre la morfología y la función que presentan, la utilización de una clasificación morfológica ha sido propuesta recientemente para el muestreo en el campo de los componentes de la costra biológica (Eldridge \& Rosentreter 1999, Belnap et al. 2001). En este estudio se utiliza la clasificación morfológica de Eldridge \& Rosentreter (1999) para identificar a los distintos componentes de la costra biológica. Entre las categorías establecidas por estos autores, dos son las más abundantes en la zona de estudio y se incluyen en este trabajo: musgos y líquenes foliacéos. Los musgos tienen un aspecto de alfombra pilosa de color verde-marrón, y están dominados por Weissia sp. La segunda está constituída por líquenes en forma de hoja que se encuentran escasamente adheridos al sustrato, siendo la especie que domina este grupo Cladonia convoluta (Lam.) P. Cout.

Para evaluar el efecto de S. tenacissima en la distribución de los musgos y líquenes foliáceos se utilizaron dos microambientes: esparto y claro. El microambiente esparto se encuentra situado en la cara norte de las macollas de S. tenacissima (a menos de $15 \mathrm{~cm}$ del borde de la macolla), mientras que el claro se localiza en las zonas desnudas sin vegetación situadas entre macollas (véase Maestre et al. 2001 para una descripción detallada). Durante la primavera de 2000 , se establecieron aleatoriamente seis cuadrados de muestreo de $50 \times 50 \mathrm{~cm}\left(0,25 \mathrm{~m}^{2}\right)$ por microambiente y parcela. Cada cuadrado se dividió en 100 subcuadrados de $5 \times 5 \mathrm{~cm}$, donde se estimó la cobertura de los mugos y los líquenes foliáceos siguiendo la escala de Braun-Blanquet: $0=0 \%, 1=1-5 \%, 2=6$ $25 \%, 3=26-50 \%, 4=51-75 \%$ y $5=76-100 \%$ (Van der Maarel 1979).

\section{Análisis del patrón espacial para cada especie}

Para caracterizar el patrón espacial de los componentes de la costra biológica por separado se ha utilizado el análisis espacial mediante índices de distancia ("spatial analysis by distance indices" o SADIE, véase Perry 1998 y Perry et al. 1999 para una descripción completa del método). Aunque desarrollada inicialmente para el análisis espacial de datos ecológicos en forma de conteos, puede utilizarse también para analizar datos en una escala ordinal (Maestre \& Cortina 2002), binaria (Perry 1998, Maestre \& Cortina 2002) o continua si se ha categorizado previamente (Perry et al. 1999). Esta técnica se basa en la "distancia a la regularidad" $(D)$, que evalua la distancia que 
los valores de la variable bajo estudio, cobertura en este caso, necesitarían moverse en el espacio para obtener una distribución regular donde la cobertura sea la misma en todos los puntos de muestreo (Perry 1995, 1998). El valor de $D$ se obtiene utilizando un algoritmo de transporte que se basa en la existencia de unidades muestrales "donantes" (con cobertura superior a la media) y "receptoras" (con cobertura inferior a la media). Para estimar la magnitud de $D$, su valor es comparado con los resultados de un test de Monte Carlo, basado en una serie de permutaciones donde los valores de cobertura son distribuidos al azar entre los puntos de muestreo (Legendre \& Legendre 1998). En particular, la división del valor observado de $D$ por el valor medio obtenido a partir de las permutaciones produce un índice de agregación, $I_{a}$, que es utilizado para describir el patrón espacial de los datos. Usualmente, los datos muestran una distribución agregada si $I_{a}>1$, aleatoria si $I_{a}=1$ y regular si $I_{a}<1$ (Perry 1998). La comparación del valor observado de $D$ con las colas de la distribución de los valores obtenidos con las permutaciones permite establecer su significación estadística (Perry et al. 1999).

Otro índice complementario proporcionado por SADIE es el índice de agrupación ( $v)$, que específicamente mide el grado de agrupación de los datos en manchas (zonas con cobertura superior a la media) y claros (zonas con cobertura inferior a la media). A su vez, este índice cuantifica el grado en el que cada unidad de muestreo contribuye al patrón espacial global en los datos. Unidades muestrales situadas dentro de una mancha tienen valores de $v$ (por convención $v_{i}$ ) elevados, mientras que aquellas que forman un claro presentan valore de $v$ (por convención $v_{j}$ ) elevados y negativos. La presencia de agrupaciones en los datos viene dada por valores elevados de $v_{i} \mathrm{o}$ $v_{j}$. Valores de $v_{i}$ mayores que 1,5 indican la presencia de una mancha, mientras que valores de $v_{j}$ inferiores $\mathrm{a}-1,5$ indican la existencia de un claro; aquellos valores cercanos a 1 indican una distribución aleatoria de esa unidad (Perry et al. 1999). Para evaluar estadísticamente el grado de agrupación de los datos, se realiza un test de Monte Carlo para unidades donantes y receptoras, respectivamente. En cada caso, los valores medios de $v_{i}$ y $v_{j}$, denominados como $V_{i}$ y $V_{j}$ respectivamente, son comparados con los valores obtenidos en las permutaciones, de la misma forma que se hace con $I_{a}$ (Perry et al. 1999). Una interesante propiedad de $v$ es que es una variable continua, lo que permite representar visualmente la localización y tamaño de las manchas y los claros.

El análisis espacial se realizó por separado para los musgos y líquenes en cada cuadrado de muestreo de 50 x $50 \mathrm{~cm}$ con el software descrito en Perry et al. (1999) y 2.340 permutaciones, utilizando como datos los valores de cobertura (0-5) obtenidos en los subcuadrado de $5 \times 5 \mathrm{~cm}$. El porcentaje de cuadrados de muestreo de 50 × 50 cm que mostró un patrón espacial no aleatorio en los dos microambientes fue analizado mediante modelos log-lineales jerarquizados (Agresti 1990). En estos análisis, los datos fueron comparados en una tabla de tres vías (significación, microambiente y parcela), utilizando un modelo no saturado (Agresti 1990). Los índices proporcionados por $\operatorname{SADIE}\left(I_{a}, V_{i}\right.$ y $\left.V_{j}\right)$ se analizaron utilizando un ANOVA de dos factores: microambiente (fijo) y parcela (aleatorio). Con el fin de corregir desviaciones respecto a la homegeneidad de varianzas, estos índices fueron transformados mediante la función logaritmo (utilizando el valor absoluto en el caso de $V_{j}$ ). Los mapas del índice de agrupación se realizaron mediante interpolación lineal con el programa SURFER (Golden Software, Colorado, USA). Para el resto de análisis estadísticos se utilizó el programa SPSS para Windows 9.0 (SPSS Inc., Chicago, Illinois, USA).

Análisis de la asociación espacial entre los musgos y líquenes

Una medida de asociación espacial debería cumplir dos requisitos principales (Perry \& Dixon 2002): (i) estar basada en una comparación clara de las propiedades espaciales de cada conjunto de datos a comparar, de modo que aquellas características del patrón espacial que coinciden en ambos contribuirían a su asociación, mientras que en caso contrario favorecerían su disociación y (ii) tener en cuenta el patrón espacial de cada conjunto de datos a comparar. La utilización del coeficiente de correlación con los valores de $v$ para los dos componentes de la costra biológica bajo estudio satisface ambas propiedades. Aquellas unidades muestrales donde los índices $v$ de ambos muestren una mancha o un claro contribuirán de manera importante y positiva a la correlación, mientras que donde una de las especies muestre una mancha y la otra un claro, la contribución será negativa. Unidades de muestreo donde los valores de $v$ sean pequeños no contribuirán de manera decisiva a la asociación o disociación. La correlación de los valores de $v$ también cumple (ii), ya que en su formación se ha tenido en cuenta el patrón espacial de cada uno de los componentes.

Debido a que la distribución de $v$ no siguió una distribución normal (prueba de Kolmogorov- 
Smirnoff; $\mathrm{P}<0,001$ en todos los casos), la significación de las correlaciones se estimó mediante un test de permutaciones (Legendre \& Legendre 1998). La significación obtenida fue ajustada para tener en consideración la autocorrelación espacial presente en los datos (Dutilleul 1993). Para ello, después de la estimación del coeficiente de correlación $(r)$, se obtienen su significación estadística y los intervalos de confianza mediante un test de permutaciones. A continuación, se realiza un análisis de tendencias superficiales ("trend surface analysis", Legendre \& Legendre 1998), consistente en una regresión entre los valores de $v$ y los nueve monomios resultantes de una combinación cúbica de las coordenadas geográficas centradas en sus correspondientes medias. Los residuales de esta regresión se sometieron al procedimiento de Dutilleul (1993) para estimar $M$, el tamaño muestral efectivo que tiene en cuenta el grado de autocorrelación presente en los datos. Los intervalos de confianza obtenidos en el test de permutaciones y su significación se ajustan multiplicándolos por $[(n-3) /(M-3)]^{1 / 2}$, donde $n$ es el número de puntos de muestreo (Perry \& Dixon 2002).

Otro tipo de información de interés que puede obtenerse con SADIE es una medida de asociación local. Este análisis permite revelar características de los datos que no son evidentes cuando se evalúa su correlación global (Anselin 1995). Con SADIE este análisis se realiza estimando la contribución de cada unidad muestral al coeficiente de correlación global entre los musgos y líquenes. En la práctica, si los índices $v$ de los líquenes foliáceos se denominan $v_{l}$, con media $q_{l}$, y los de los musgos $v_{2}$, con media $q_{2}$, una medida de la asociación espacial para la unidad muestral $i\left(c_{i}\right)$ se obtiene mediante la siguiente relación (Winder et al. 2001, Perry \& Dixon 2002):

$$
\chi_{\mathrm{i}}=\frac{n\left(v_{i 1}-q_{1}\right)\left(v_{i 2}-q_{2}\right)}{\sqrt{\sum_{i}\left(v_{i 1}-q_{1}\right)^{2}\left(v_{i 2}-q_{2}\right)^{2}}}
$$

donde $n$ es el número de unidades muestrales (100 en este caso). Valores positivos de $\chi$ indican la presencia de coincidencias en las manchas y/o claros, mientras que valores negativos revelan la presencia de una mancha en un componente de la costra y un claro en el otro. Es interesante destacar el hecho de que la media de $\chi$ equivale al coeficiente de correlación. Los valores de $\chi$ son continuos y están autocorrelados, por lo que se pueden representar en un mapa en dos dimensiones con el fin de visualizar las zonas donde se produce asociación o disociación dentro de cada cuadrado muestreado. Un intervalo de confianza para esta medida puede obtenerse a partir del test de permutaciones utilizado para evaluar la correlación global entre los dos componentes de la costra biológica evaluados. Para ello se multiplica el intervalo de confianza obtenido durante dicho análisis por la raíz cuadrada de $n$ (Perry \& Dixon 2002).

Los análisis de correlaciones y asociación local se realizaron para cada cuadradado de muestreo de 50 x $50 \mathrm{~cm}$ con el software descrito en Perry \& Dixon (2002). Los mapas de asociación local se crearon con el software Surfer para Windows 5.0 (Golden Software, Boulder, Colorado, USA).

\section{RESULTADOS}

Los líquenes foliáceos mostraron por lo general un patrón espacial no aleatorio, ya que el $100 \%$ y el 67-83\% de los cuadrados muestreados en los microambientes esparto y claro, respectivamente, presentaron valores de $I_{a}$ significativamente diferentes que uno (Tabla 1). Su patrón espacial estuvo afectado por el microambiente, ya que el número de cuadrados muestreados donde el valor de $I_{a}$ fue estadísticamente diferente de uno fue superior en el esparto (análisis log-lineal; $\left.G^{2}{ }_{\text {Microambiente x Ia } \neq 1}=7,83 ; \mathrm{g} .1 .=1 ; \mathrm{P}=0,005\right)$. Los índices $I_{a}, V_{i}$ y $V_{j}$ (valores absolutos en este último caso) obtenidos para este componente de la costra biológica fueron siempre superiores a 1,3, sugiriendo la presencia de un patrón de distribución agregado (Tabla 2). Todos ellos mostraron valores significativamente superiores (excepto en $V_{j}$, que fueron inferiores) en el microambiente esparto respecto al claro (Tabla 3). En el caso de los musgos, el número de cuadrados muestreados con un patrón espacial no aleatorio fluctuó entre 67$83 \%$ en el microambiente esparto y 50-67\% en el claro, aunque estas diferencias no fueron significativas (análisis log-lineal, $G^{2}{ }_{\text {Microambiente x } I a \neq 1}=$ 2,$21 ;$ g.l. $=1 ; \mathrm{P}=0,137$ ). Los valores medios obtenidos para $I_{a}$ y $v_{i}$ fueron en general inferiores a los obtenidos para los líquenes foliáceos (Tabla 2). A pesar de que se aprecia una mayor agregación en el microambiente esparto, no se encontraron diferencias significativas entre microambientes para ninguno de los índices SADIE en los musgos (Tabla 3).

Los mapas de $v$ mostraron la distribución de las manchas y los claros dentro de los cuadrados muestreados (Fig. 1). De su observación pueden apreciarse dos características principales en la distribución espacial de los líquenes foliáceos: un mayor nivel de agregación en el microambiente esparto respecto al claro y una disminución de 
TABLA 1

Frecuencia de cuadrados de muestreo que mostraron un patrón espacial no aleatorio $\left(I_{a} \neq 1 ; \mathrm{P}\right.$ $<0,05)$. Para cada combinación de parcela, microambiente y componente de la costra biológica, $\mathrm{n}=6$

Frequency of sampled squares that showed a non-random spatial pattern $\left(I_{a} \neq 1, \mathrm{P}<0.05\right)$. For all combinations of site, microsite and biological crust component, $\mathrm{n}=6$

Componente de la costra biológica

\begin{tabular}{lcccccc} 
& \multicolumn{2}{c}{ Colominas } & \multicolumn{2}{c}{ La Cueva } & \multicolumn{2}{c}{ La Torre } \\
& Esparto & Claro & Esparto & Claro & Esparto & Claro \\
\hline Líquenes foliáceos & 100 & 83 & 100 & 67 & 100 & 67 \\
Musgos & 83 & 67 & 67 & 50 & 83 & 67 \\
\hline
\end{tabular}

ésta en el primero conforme aumenta la distancia al borde de $S$. tenacissima. En el microambiente claro, esta tendencia hacia la agregación en los alrededores de $S$. tenacissima no fue tan clara. Los musgos presentaron un comportamiento similar, aunque la mayor variabilidad entre cuadradados propició la falta de diferencias significativas entre microambientes. El número de cuadrados donde el patrón espacial de ambas especies mostró una relación significativa no mostró diferencias entre microambientes (Tabla 4, Análisis log-lineal, $G^{2}$

Microambientex Correlación significativa reflejó claramente en los mapas de asociación local (Fig. 2).
DISCUSIÓN

Los resultados obtenidos apoyan la hipótesis inicial de que el microambiente proporcionado por $S$. tenacissima es capaz de promover cambios en la distribución espacial de los componentes de la costra biológica, aunque no en la covariación espacial entre ellos. No obstante, los componentes de esta costra analizados mostraron una respuesta diferencial al microambiente, ya que el patrón espacial de los musgos no se vio afectado de forma significativa por la presencia de $S$. tenacissima.

Los líquenes foliáceos mostraron un mayor grado de agregación en el microambiente esparto,

TABLA 2

Resumen del análisis SADIE para los componentes de la costra biológica evaluados. Los datos representan la media \pm el error estándar $(\mathrm{n}=6) ; I_{a}=$ índice de agregación, $V_{j}=$ media del índice de agrupación para los claros, $V_{i}=$ media del índice de agrupación para las manchas

Summary of SADIE indices obtained for the biological crusts components evaluated. Data represent mean \pm 1 SE (n $=6) ; I_{a}=$ index of aggregation, $V_{i}=$ mean of the index of clustering for patches, and $V_{j}=$ mean of the index of clustering for gaps

\begin{tabular}{lllccc}
\hline Parcela & Componente & Microambiente & $I_{a}$ & $V_{i}$ & $V_{j}$ \\
\hline Colominas & \multirow{2}{*}{ Líquenes foliáceos } & Esparto & $2,81 \pm 0,15$ & $2,55 \pm 0,55$ & $-2,66 \pm 0,14$ \\
& & Claro & $1,55 \pm 0,11$ & $1,48 \pm 0,08$ & $-1,55 \pm 0,11$ \\
& \multirow{4}{*}{ La Cueva } & Esparto & $1,74 \pm 0,26$ & $1,67 \pm 0,22$ & $-1,65 \pm 0,21$ \\
& \multirow{4}{*}{ Líquenes foliáceos } & Claro & $1,55 \pm 0,25$ & $1,35 \pm 0,20$ & $-1,49 \pm 0,23$ \\
& & Esparto & $2,94 \pm 0,29$ & $2,65 \pm 0,33$ & $-2,71 \pm 0,26$ \\
& \multirow{3}{*}{ La Torre } & Claro & $1,42 \pm 0,12$ & $1,43 \pm 0,13$ & $-1,39 \pm 0,11$ \\
& \multirow{3}{*}{ Líquenes foliáceoso } & Esparto & $1,50 \pm 0,17$ & $1,42 \pm 0,14$ & $-1,57 \pm 0,17$ \\
& & Claro & $1,43 \pm 0,21$ & $1,40 \pm 0,21$ & $-1,40 \pm 0,22$ \\
& \multirow{2}{*}{ Musgo } & Esparto & $2,53 \pm 0,32$ & $2,40 \pm 0,34$ & $-2,35 \pm 0,31$ \\
& & Claro & $1,55 \pm 0,16$ & $1,56 \pm 0,16$ & $-1,54 \pm 0,16$ \\
& Esparto & $2,52 \pm 0,30$ & $2,32 \pm 0,27$ & $-2,40 \pm 0,29$ \\
& & Claro & $1,63 \pm 0,22$ & $1,60 \pm 0,22$ & $-1,52 \pm 0,21$ \\
\hline
\end{tabular}




\section{Liquenes foliáceos}
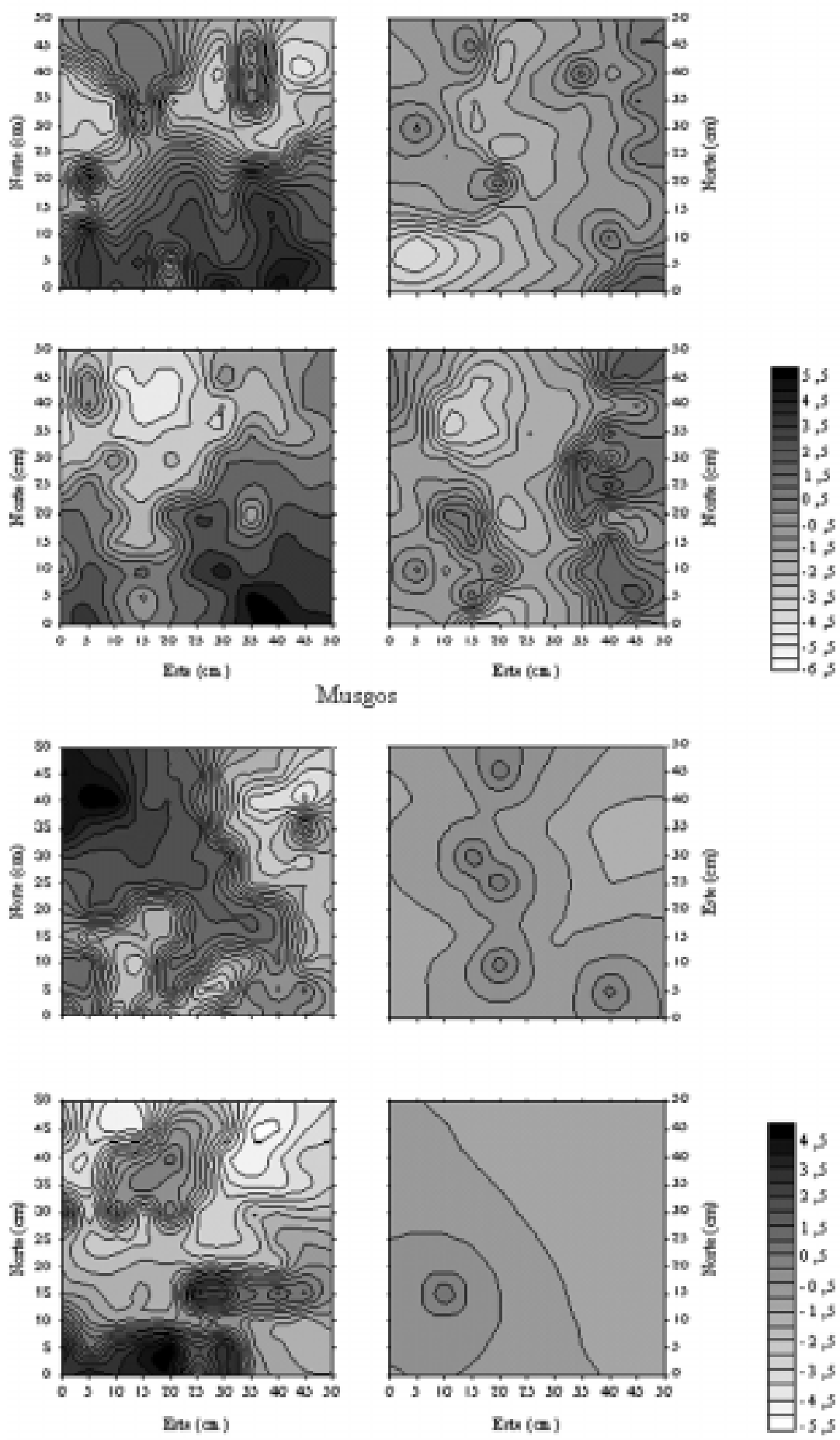

Fig. 1: Mapas del índice de agrupación (v) de los líquenes foliáceos y los musgos obtenidos en los microambientes esparto (izquierda) y claro (derecha). En el esparto, el eje norte refleja la distancia desde el borde de Stipa tenacissima. Por brevedad, sólo se muestran algunos mapas obtenidos en la parcela Colominas. La leyenda no tiene unidades.

Contour maps of SADIE index of clustering for foliose lichens and mosses in tussock (left) and open (right) microsites. In the tussock microsite, the north axis reflects the distance from the edge of the tussock. For brevity, only selected maps from the Colominas site are shown. The legend has no units. 

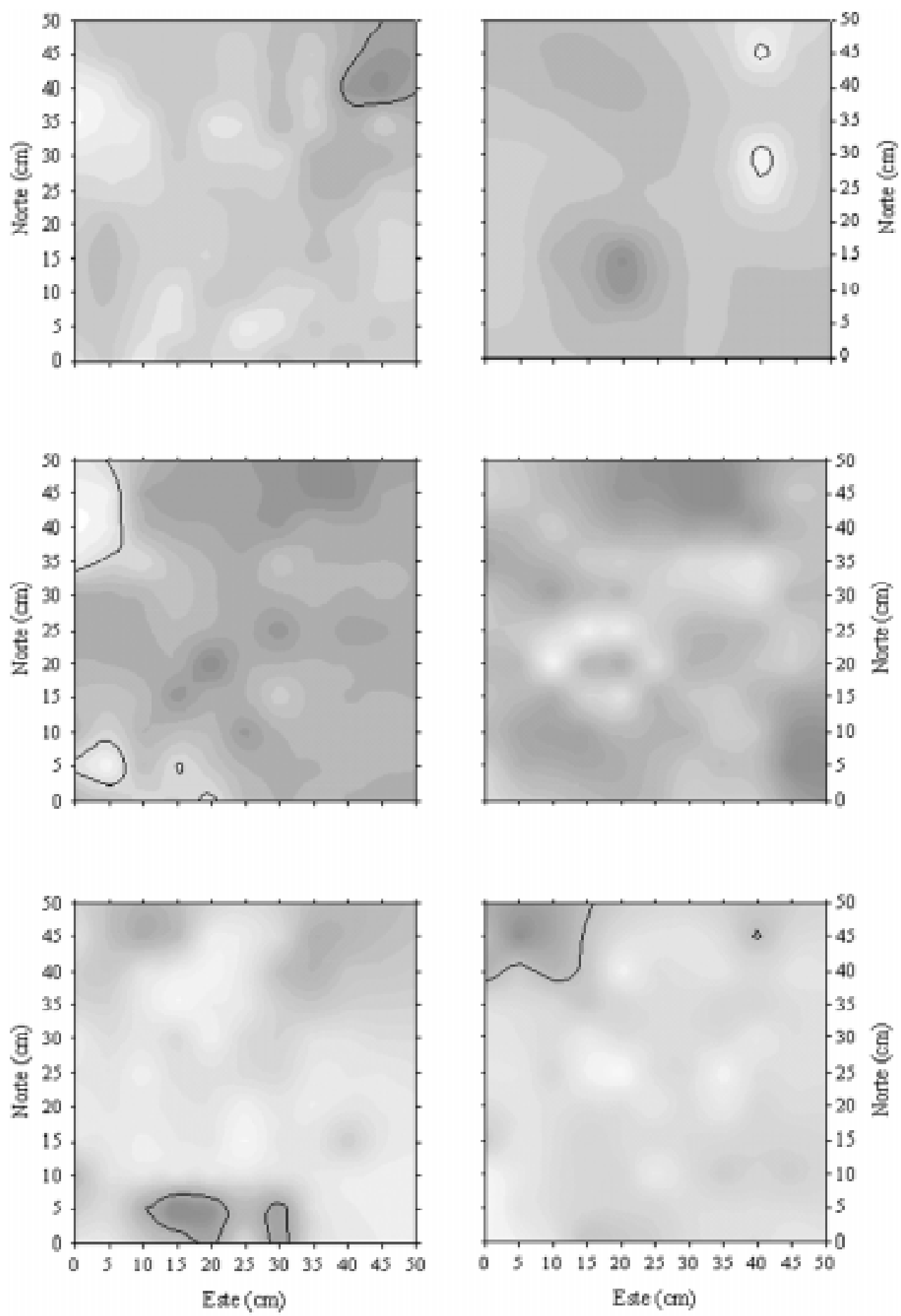

Fig. 2: Mapas de asociación local entre los líquenes foliáceos y los musgos en el microambiente esparto (izquierda) y claro (derecha). Las zonas de color blanco y gris indican una asociación espacial negativa y positiva respectivamente. Las zonas de asociación significativa $(\mathrm{P}<0,05)$ están rodeadas en negro. Por brevedad, sólo se muestran algunos mapas obtenidos en la parcela Colominas.

Contour maps of local association between foliose lichens and mosses in tussock (left) and open (right) microsites. White and grey patches represent areas of negative and positive association, respectively. Significant positive or negative association areas $(\mathrm{P}<0.05)$ are included within a solid line. In the tussock microsite the north axis reflects the distance from the edge of the tussock. For brevity, only selected maps from the Colominas site are shown. 
TABLA 3

Resultados de un ANOVA (valores de F, P y grados de libertad, g.1.) de dos factores donde se evalúa el efecto del microambiente y la parcela en los valores de los índices SADIE

(transformados mediante una función logaritmo); $I_{a}=$ índice de agregación, $V_{j}=$ media del índice de agrupación para los claros y $V_{i}=$ media del índice de agrupación para las manchas

Results of a two-way ANOVA (F, P value and degrees of freedom, g.1.) to assess the significance of the microsite and site effect on log-transformed SADIE indices; $I_{a}=$ index of aggregation, $V_{i}=$ mean of the index of clustering for patches, and $V_{j}=$ mean of the index of clustering for gaps

\begin{tabular}{|c|c|c|c|c|c|c|c|c|c|c|}
\hline \multirow{3}{*}{ Componente } & \multirow{3}{*}{$\begin{array}{l}\text { Índice } \\
\text { SADIE }\end{array}$} & \multicolumn{9}{|c|}{ Resultados del ANOVA } \\
\hline & & \multicolumn{3}{|c|}{ Microambiente (M) } & \multicolumn{3}{|c|}{ Parcela (P) } & \multicolumn{3}{|c|}{$\mathrm{M} \times \mathrm{P}$} \\
\hline & & $\mathrm{F}$ & g.1. & $\mathrm{P}$ & $\mathrm{F}$ & g.1. & $\mathrm{P}$ & $\mathrm{F}$ & g.1. & $\mathrm{P}$ \\
\hline \multirow[t]{3}{*}{ Líquenes foliáceos } & $I_{a}$ & 76,13 & 1,2 & 0,013 & 0,37 & 2,2 & 0,730 & 0,761 & 2,30 & 0,476 \\
\hline & $\stackrel{a}{V_{i}}$ & 91,57 & 1,2 & 0,011 & 0,17 & 2,2 & 0,854 & 0,398 & 2,30 & 0,675 \\
\hline & $V_{j}^{l}$ & 55,72 & 1,2 & 0,017 & 0,53 & 2,2 & 0,654 & 0,843 & 2,30 & 0,440 \\
\hline \multirow[t]{3}{*}{ Musgos } & $I_{a}^{J}$ & 3,43 & 1,2 & 0,205 & 2,66 & 2,2 & 0,273 & 0,96 & 2,30 & 0,395 \\
\hline & $\stackrel{a}{V_{i}}$ & 4,83 & 1,2 & 0,159 & 3,71 & 2,2 & 0,212 & 0,84 & 2,30 & 0,442 \\
\hline & $V_{j}^{l}$ & 4,78 & 1,2 & 0,160 & 2,00 & 2,2 & 0,333 & 0,93 & 2,30 & 0,407 \\
\hline
\end{tabular}

donde formaron manchas continuas que se volvieron más dispersas conforme aumentó la distancia al borde de $S$. tenacissima. En el microambiente claro, las extensiones ocupadas por este componente de la costra biológica fueron más pequeñas y dispersas, quedando claramente reflejadas las diferencias entre microambientes en los valores de $I_{a}$. Los resultados obtenidos sugieren que $S$. tenacissima es capaz de facilitar el desarrollo de los líquenes foliáceos (sensu Callaway 1995) y coinciden con descripciones generales de este componente de la costra biológica en zonas semiáridas (Belnap et al. 2001). A pesar de que Cladonia convoluta, el principal constituyente de este grupo morfológico en la zona de estudio, es capaz de retener el contenido en clorofila y su maquinaria fotosintética durante los períodos de desecación (Tuba et al. 1996), este proceso convierte al fotosistema II en un estado no funcional (Sass et al. 1996). Además, esta especie presenta una acusada pérdida de rigidez en las paredes celulares cuando su contenido relativo en agua disminuye por debajo del $60 \%$ (Proctor et al. 1998). Estos cambios fisiológicos limitan notablemente su actividad fotosintética y crecimiento, por lo que la mejora en las condiciones microclimáticas promovida por el desarrollo de $S$. tenacissima parece ser el principal mecanismo implicado en la facilitación encontrada.

La falta de diferencias significativas en el patrón espacial de los musgos entre microambientes fue inesperada, ya que la mejora de las condiciones microclimáticas promovida por $S$. tenacissima (Maestre et al. 2001) debería propiciar un mayor desarrollo de los musgos -limitado en zonas semiáridas por la escasez de precipitaciones y la baja humedad ambiental- en los alrededores de esta especie. No obstante, se aprecia una cierta

TABLA 4

Frecuencia de cuadrados de muestreo donde el patrón espacial de componentes de la costra biológica evaluados mostró una relación significativa $(\mathrm{P}<0,05)$. Para combinación de parcela y microambiente, $n=6$

Frequency of quadrats where the correlation coefficient showed a significant relationship $(\mathrm{P}<0.05)$ between the components of biological crusts evaluated. For all combinations of site and microsite, $n=6$

\begin{tabular}{cccccc}
\hline & & \multicolumn{2}{c}{$\begin{array}{c}\text { Frecuencia }(\%) \\
\text { La Cueva }\end{array}$} & \multicolumn{2}{c}{ La Torre } \\
\hline $\begin{array}{c}\text { Colominas } \\
\text { Esparto }\end{array}$ & Claro & Esparto & Claro & Esparto & Claro \\
50 & 33 & 50 & 67 & 50 & 67 \\
\hline
\end{tabular}


tendencia a encontrar una mayor agregación de los musgos en los alrededores de S. tenacissima. Las observaciones recogidas en este estudio concuerdan con las de Maestre \& Cortina (2002), que no detectan una asociación positiva y significativa entre el patrón espacial de $S$. tenacissima y los musgos en una parcela de 50 x 50 m localizada a 60 $\mathrm{km}$ de la zona de estudio. No obstante, contrastan con estudios llevados a cabo a escala de individuo, que detectan una mayor cobertura de musgos en el microambiente esparto respecto al claro (MartínezSánchez et al. 1994, Maestre et al. 2002).

Los análisis realizados no apoyan la existencia de interacciones bióticas entre los líquenes foliáceos y los musgos en los espartales estudiados. La naturaleza descriptiva del presente estudio no permite establecer los mecanismos implicados en los patrones de variación conjunta observados, por lo que nuevas experiencias son necesarias para establecer sus causas y analizar su dinámica temporal. En este sentido, y debido al lento crecimiento que caracteriza a los componentes de la costra biológica, no se espera la existencia de grandes cambios en las asociaciones encontradas a cortomedio plazo. No obstante, podrían verse alteradas por las modificaciones climáticas previstas para las próximas décadas, que apuntan hacia un incremento de la severidad y recurrencia de los periodos de sequía en la parte oriental de la cuenca mediterránea (Cubasch et al. 1996, De Luis et al. 2001). Este endurecimiento de las condiciones ambientales podría hacer más dependiente aún la distribución de los musgos y líquenes de la mejora de las condiciones microclimáticas asociada a $S$. tenacissima, lo que podría modificar las interacciones entre ambos al aumentar la competencia por el espacio en el microambiente esparto.

Las diferencias encontradas entre microambientes en la distribución de los líquenes foliáceos y, en menor medida, la tendencia que presentan los musgos a desarrollarse principalmente en las cercanías de las macollas de $S$. tenacissima, pueden tener notables influencias en los flujos hidrológicos de los espartales donde estas especies son abundantes. Si bien el papel de los líquenes en la generación de escorrentía y en la infiltración no está del todo claro y parece depender fuertemente de las condciones ambientales específicas de cada lugar (Eldridge \& Greene 1994, Belnap et al. 2001), el incremento en la rugosidad de la superficie edáfica promovido por el desarrollo de este componente de la costra biológica puede aumentar la tasa de infiltración y actuar como una trampa para las semillas de especies vasculares (Zaady et al. 1997). Por su parte, los musgos son capaces de absorber grandes cantidades de agua después de los eventos lluviosos y de aumentar la infiltración (Maestre et al. 2002), así como de retener esta humedad en superficie durante períodos de tiempo considerables (Sharrat 1997, Eldridge \& Rosenter 1999, Belnap et al. 2001). Así pues, es posible que el mayor desarrollo de estos componentes de la costra biológica en las cercanías de $S$. tenacissima pueda incrementar la infiltración y la captura de semillas, contribuyendo así a crear un microambiente propicio para el desarrollo de las plantas vasculares (Sánchez 1995, Maestre et al. 2001). Numerosos estudios realizados en zonas templadas han puesto de manifiesto como la presencia de musgos en superficie proporciona un microambiente favorable para la germinación y el establecimiento de numerosas especies leñosas (e.g., Hilaire-Lisa \& Leopold 1995, Parker et al. 1997, Kameyama et al. 1999). No obstante, poco se conoce sobre su efecto en el establecimiento de plantas vasculares bajo condiciones ambientales semiáridas (Eldridge \& Greene 1994), por lo que es necesario realizar nuevos estudios para evaluar su papel en este proceso, especialmente importante para la dinámica de la vegetación en ambientes mediterráneos (Jordano 2001).

SADIE detectó la presencia de patrones espaciales agregados en los componentes de la costra biológica evaluados. Entre las principales virtudes de SADIE se encuentran su simplicidad de uso, su sencilla base matemática y la utilización de tests estadísticos para evaluar la significación de los patrones espaciales observados. Además, los mapas de $v$ reflejan la localización espacial y dimensiones de las manchas y los claros, proporcionando información visual complementaria que puede facilitar la interpretación de los resultados (Diggle 1983). Otras características de interés que presenta esta metodología frente a otras existentes son el hecho de que tiene en cuenta la localización espacial de los datos (Perry 1998), éstos no necesitan seguir ninguna distribución determinada y los resultados obtenidos están condicionados únicamente por su heterogeneidad (Bell 1998). SADIE ha permitido caracterizar de manera satisfactoria la distribución espacial de los componentes de la costra biológica evaluados, habiéndose mostrado como una herramienta válida para su análisis cuando los datos son tomados en una escala binaria o semi-cuantitativa, tal como ocurre frecuentemente en estudios florísticos y ecológicos en los que intervienen estos organismos (Eldridge 1996, Eldridge \& Tozer 1997, Eldridge \& Koen 1998).

Si bien el análisis de la distribución espacial de los constituyentes de la costra biológica ha recibido poca atención (Dale 1995), su elucidación permite obtener valiosa información sobre su utilización de recursos ambientales, las interacciones 
bióticas entre ellos y sus relaciones con otros organismos (Dale 1999). Los resultados obtenidos contribuyen al conocimiento de las interacciones entre las plantas vasculares y la costra biológica en zonas semiáridas. Las distribuciones encontradas pueden tener importantes implicaciones en la generación y dinámica de los flujos de agua, nutrientes y sedimentos en los espartales, así como en las interacciones entre las distintas especies vegetales, influenciando así en el funcionamiento de estos ecosistemas. Nuevos estudios son necesarios para establecer el papel de los patrones espaciales encontrados en estos procesos.

\section{AGRADECIMIENTOS}

Estoy especialmente agradecido a Joe Perry por su ayuda con el método SADIE y por facilitarme todo el software necesario para poder realizar los análisis presentados en este trabajo, así como a María Dolores Puche por su colaboración en la toma de datos de campo y a dos revisores anónimos por las sugerencias y comentarios aportados. Este trabajo ha sido financiado por una beca FPU del Ministerio de Educación, Cultura y Deporte español, así como por el proyecto REDMED, financiado por la Comisión Europea.

\section{LITERATURA CITADA}

AGUIAR MR \& OE SALA (1999) Patch structure, dynamics and implications for the functioning of arid ecosystems. Trends in Ecology and Evolution 14: 273-277.

AGRESTI A (1990) Categorical data analysis. John Wiley $\&$ Sons, New York, New York. 576 pp.

ANSELIN L (1995) Local indicators of spatial associationLISA. Geographical Analysis 27: 93-115.

BELL ED (1998) Spatio-temporal dynamics of UK moths. Tesis Doctoral, Universidad de Leicester, Leicester, United Kingdom. 278 pp.

BELNAP J, JH KALTENECKER, R ROSENTRETER, J WILLIAMS, S LEONARD \& D ELDRIDGE (2001) Biological soil crusts: ecology and management. U.S. Department of the Interior, Bureau of Land Management, National Science and Technology Center. Denver, Colorado. v + 110 pp.

BINKLEY D \& C GIARDINA (1998) Why do tree species affect soils? The warp and woof of tree-soil interactions. Biogeochemistry 42: 89-106.

BOCHET E, JL RUBIO \& J POESEN (1999) Modified topsoil islands within patchy Mediterranean vegetation in SE Spain. Catena 38: 23-44.

BRESHEARS DD, JW NYHAN, CE HEIL \& BP WILCOX (1998) Effects of woody plants on microclimate in a semiarid woodland: soil temperature and evaporation in canopy and intercanopy patches. International Journal of Plant Sciences 159: 1010-1017.
CALLAWAY RM (1995) Positive interactions among plants. Botanical Review 61: 306-349.

CERDÀ A (1997) The effect of patchy distribution of Stipa tenacissima $\mathrm{L}$. on runoff and erosion. Journal of Arid Environments 36: 37-51.

CROSS AF \& WH SCHLESINGER (1999) Plant regulation of soil nutrient distribution in the northern Chihuahuan Desert. Plant Ecology 145: 11-25.

CUBASCH U, H VON STORCH, J WASZKEWITZ \& E ZORITA (1996) Estimates of climate change in southern Europe derived from dynamical climate model output. Climate Research 7: 129-149.

DALE MRT (1995) Spatial pattern in communities of crustose saxicolous lichens. Lichenologist 27: 495503.

DALE MRT (1999) Spatial pattern analysis in plant ecology. Cambridge University Press, Cambridge, United Kingdom. 326 pp.

DEFALCO LA, JK DETLING, C RICHARD TRACY \& SD WARREN (2001) Physiological variation among native and exotic winter annual plants associated with microbiotic crusts in the Mojave Desert. Plant and Soil 234: 1-14.

DE LUIS M, MF GARCÍA-CANO, J CORTINA, J RAVENTÓS, JC GONZÁLEZ-HIDALGO \& JR SÁNCHEZ (2001) Climatic trends, disturbances and short-term vegetation dynamics in a Mediterranean shrubland. Forest Ecology and Management 147: 2537.

DIGGLE PJ (1983) Statistical analysis of spatial point patterns. Academic Press, London, United Kingdom. $183 \mathrm{pp}$.

DUTILLEUL P (1993) Modifying the t test for assessing the correlation between two spatial processes. Biometrics 49: 305-314.

ELDRIDGE DJ (1996) Distribution and floristics of terricolous lichens in soil crusts in arid and semi-arid New South Wales, Australia. Australian Journal of Botany 44: 581-599.

ELDRIDGE DJ \& RSB GREENE (1994) Microbiotic soil crusts: a review of their roles in soil and ecological processes in the rangelands of Australia. Australian Journal of Soil Research 32: 389-415.

ELDRIDGE DJ \& ME TOZER (1997) Environmental factors relating to the distribution of terricolous byrophytes and lichens in semi-arid eastern Australia. Bryologist 100: 28-39.

ELDRIDGE DJ \& TB KOEN (1998) Cover and floristics of microphytic soil crusts in relation to indices of landscape health. Plant Ecology 137: 101-114.

ELDRIDGE DJ \& R ROSENTRETER (1999) Morphological groups: a framework for monitoring microphytic crusts in arid landscapes. Journal of Arid Environments 41: 11-25.

ELDRIDGE DJ, E ZAADY \& M SHACHAK (2000) Infiltration through three contrasting biological soil crusts in patterned landscapes in the Negev, Israel. Catena 40: 323-336.

HARPER KT \& J BELNAP (2001) The influence of biological soil crusts on mineral uptake by associated vascular plants. Journal of Arid Environments 47: 347-357.

HILAIRE-LISA RS \& DJ LEOPOLD (1995) Conifer seedling distribution in relation to microsite conditions in a central New York forested minerotrophic peatland. Canadian Journal of Forest Research 25: 261-269. 
JORDANO P (2001) Conectando la ecología de la reproducción con el reclutamiento poblacional de plantas leñosas mediterráneas. En: Zamora R \& FI Pugnaire (eds) Ecosistemas mediterráneos. Análisis funcional: 183-212. Asociación Española de Ecología Terrestre y Consejo Superior de Investigaciones Científicas, Madrid, España.

KAMEYAMA Y, N NAKAGOSHI \& K NEHIRA (1999) Safe site for seedlings of Rhododendron metternichii var. hondoense. Plant Species Biology 14: 237-242.

LANGE OL, GJ KIDRON, B BUEDEL, A MEYER, E KILIAN \& A ABELIOVICH (1992) Taxonomic composition and photosynthetic characteristics of the "biological soil crusts" covering sand dunes in the western Negev Desert. Functional Ecology 6: 519-527.

LEGENDRE P \& L LEGENDRE (1998) Numerical ecology. Second edition. Elsevier Publishers, Amsterdam, The Netherlands. 853 pp.

LE HOUÉROU HN (2001) Biogeography of the arid steppeland north of the Sahara. Journal of Arid Environments 48: 103-128.

MAESTRE FT (2000) El medio ambiente en Sax. Estado actual y propuestas de gestión. Universidad de Alicante y Ayuntamiento de Sax, Alicante, España. 192 pp.

MAESTRE FT \& J CORTINA (2002) Spatial pattern of soil properties and vegetation in a Mediterranean semi-arid steppe. Plant and Soil 241: 279-291.

MAESTRE FT, S BAUTISTA, J CORTINA \& J BELLOT (2001) Potential of using facilitation by grasses to establish shrubs on a semiarid degraded steppe. Ecological Applications 11: 1651-1655.

MAESTRE FT, MT HUESCA, E ZAADY, S BAUTISTA \& J CORTINA (2002) Infiltration, penetration resistance and microphytic crust composition in contrasted microsites within a Mediterranean semi-arid steppe. Soil Biology \& Biochemistry 34: 895-898.

MARTÍNEZ-SÁNCHEZ JJ, M CASARES-PORCEL, J GUERRA，L GUTIÉRREZ-CARRETERO，RM ROS，J HERNÁNDEZ-BASTIDA \& MJCANO (1994) A special habitat for bryophytes and lichens in the arid zones of Spain. Lindbergia 19: 116-121.

MATEO G \& MB CRESPO (2001) Manual para la determinación de la flora valenciana. Moliner-40, Valencia, España. $503 \mathrm{pp}$.

PARKER WC, SR WATSON \& DW CAIRNS (1997) The role of hair-cap mosses (Polytrichum spp.) in natural regeneration of white spruce (Picea glauca [Moench] Voss). Forest Ecology and Management 92: 19-28.

PERRY JN (1995) Spatial aspects of animal and plant distribution in patchy farmland habitats. En: Glen DM, MP Greaves \& HM Anderson (eds) Ecology and integrated farming systems: 95-102. John Wiley \& Sons Ltd, Bristol, United Kingdom.

PERRY JN (1998) Measures of spatial pattern for counts. Ecology 79: 1008-1017.

PERRY JN \& P DIXON (2002) A new method to measure spatial association for ecological count data. Ecoscience 9: 133-141.

PERRY JN, L WINDER, JM HOLLAND \& RD ALSTON (1999) Red-blue plots for detecting clusters in count data. Ecology Letters 2: 106-113.
PRASSE R \& R BORNKAMM (2000) Effect of microbiotic soil surface crusts on emergence of vascular plants. Plant Ecology 150: 65-75.

PROCTOR MCF, Z NASY, Z CSINTALAN \& Z TAKÁCS (1998) Water-content components in bryophytes: Analysis of pressure-volume relationships. Journal of Experimental Botany 49: 1845-1954.

PUIGDEFÁBREGAS J \& G SÁNCHEZ (1996) Geomorphological implications of vegetation patchiness on semiarid slopes. En: Anderson MG \& SM Brooks (eds) Advances in hillslope processes, Volume 2: 10271060. John Willey \& Sons, London, United Kingdom.

PUIGDEFÁBREGAS J, A SOLÉ-BENET, L GUTIÉRREZ, G DEL BARRIO \& M BOER (1999) Scales and processes of water and sediment redistribution in drylands: results from the Rambla Honda field site in southeast Spain. Earth-Science Reviews 48: 39-70.

REYNOLDS JF, RA VIRGINIA, PR KEMP, AG DE SOYZA \& DC TREMMEL (1999) Impact of drought on desert shrubs: effects of seasonality and degree of resource island development. Ecological Monographs 69: 69106.

SÁNCHEZ G (1995) Arquitectura y dinámica de las matas de esparto (Stipa tenacissima L.), efectos en el medio e interacciones con la erosion. Tesis Doctoral, Universidad Autónoma de Madrid, Madrid, España. x + 119 pp.

SASS L, Z CSINTALAN, Z TUBA \& I VASS (1996) Thermoluminiscence studies on the function of the photosystem II in the desiccation tolerant lichen Cladonia convoluta. Photosynthesis Research 48: 205-212.

SCHLESINGER WH \& AM PILMANIS (1998) Plant-soil interactions in desert. Biogeochemistry 42: 169-187.

SHARRATT BS (1997) Thermal conductivity and water retention of a black spruce forest floor. Soil Science 162: 576-582.

TUBA Z, Z CSINTALAN \& MCF PROCTOR (1996) Photosynthetic responses of a moss, Tortula ruralis, ssp. ruralis, and the lichens Cladonia convoluta and C. furcata to water deficit and short periods of desiccation, and their ecophysiological significance: a baseline study at present-day $\mathrm{CO}_{2}$ concentration. New Phytologist 133: 353-361.

TURECHECK WW \& LV MADDEN (1999) Spatial pattern of strawberry leaf blight in perennial production systems. Phytopathology 89: 421-433.

VAN DER MAAREL E (1979) Transformation of coverabundance values in phytosociology and its effects on community similarity. Vegetatio 39: 97-114.

WEST NE (1990) Structure and function of mycrophytic soil crusts in wildland ecosystems of arid to semi-arid regions. Advances in Ecological Research 20: 179-223.

WINDER L, C ALEXANDER, JM HOLLAND, C WOOLLEY \& JN PERRY (2001) Modelling the dynamic spatiotemporal response of predators to transient prey patches in the field. Ecology Letters 4: 568-576.

ZAADY E, Y GUTTERMAN \& B BOEKEN (1997) The germination of mucilaginous seeds of Plantago coronopus, Reboudia pinnata, and Carrichtera annua on cyanobacterial soil crust from the Negev Desert. Plant and Soil 190: 247-252. 